\title{
First Light of the 1.6 meter off-axis New Solar Telescope at Big Bear Solar Observatory
}

\author{
Wenda Cao ${ }^{a b}$, Nicolas Gorceix ${ }^{b}$, Roy Coulter $^{b}$, Aaron Coulter $^{b}$, Philip R. Goode ${ }^{a b}$ \\ ${ }^{a}$ Center for Solar-Terrestrial Research, New Jersey Institute of Technology, \\ 323 Martin Luther King Blvd., Newark, NJ 07102; \\ ${ }^{b}$ Big Bear Solar Observatory, 40386 North Shore Lane, Big Bear City, CA 92316
}

\begin{abstract}
New Jersey Institute of Technology, in collaboration with the University of Hawaii and the Korea Astronomy \& Space Science Institute, has successfully developed and installed a $1.6 \mathrm{~m}$ clear aperture, off-axis New Solar Telescope (NST) at the Big Bear Solar Observatory. The NST will be the largest aperture solar telescope in the world until the $4 \mathrm{~m}$ Advanced Technology Solar Telescope (ATST) and $4 \mathrm{~m}$ European Solar Telescope (EST) begin operation in the next decade. Meanwhile, the NST will be the largest off-axis telescope before the $8.4 \mathrm{~m}$ segmented Giant Magellan Telescope (GMT) comes on-line. The NST is configured as an off-axis Gregorian system consisting of a parabolic primary, prime focus field stop and heat reflector, elliptical secondary and diagonal flats. The primary mirror is made of Zerodur from Schott and figured to a final residual error of 16 $\mathrm{nm}$ rms by Steward Observatory Mirror Lab. The final focal ratio is $\mathrm{f} / 52$. The $180^{\prime \prime}$ circular opening in the field stop defines the maximal square field-of-view. The working wavelength range will cover 0.4 to $1.7 \mu \mathrm{m}$ in the Coudé Lab two floors beneath the telescope, and all wavelengths including far infrared at the Nasmyth focus on an optical bench attached to the side of the telescope structure. First-light scientific observations have been attained at the Nasmyth focus and in the Coudé Lab. This paper presents a detailed description of installation and alignment of the NST. First-light observational results are also shown to demonstrate the validity of the NST optical alignment.
\end{abstract}

Keywords: Telescope, Alignment, Wave front sensor, Solar observation

\section{INTRODUCTION}

Solar observation and research are striding into a new era of high temporal and spatial resolutions, which cannot be accomplished without large-aperture solar telescopes. It has been generally accepted that the magnetoconvection rooted on the photosphere plays a critical role in solar activity and variability. To verify and guide modeling of magneto-convection and flux emergence, transport and annihilation, meters-class solar telescopes are indispensable since these features only have a typical size of $70 \sim 100 \mathrm{~km}$. The former main telescope at the Big Bear Solar Observatory (BBSO), built in 1973, was an on-axis vacuum reflector with a clear aperture of $65 \mathrm{~cm}$. Diffraction limited resolutions was $0.2^{\prime \prime}(\sim 150 \mathrm{~km}$ on the Sun) in the visible wavelength regime and only $0.6^{\prime \prime}$ in the near infrared. Obviously, this sub-meter telescope no longer meets the need for high resolution observations. After a nearly eight-year effort, the $1.6 \mathrm{~m}$ clear aperture, off-axis New Solar Telescope (NST) was successfully developed and installed at the BBSO. The NST will be the largest aperture solar telescope in the world before the $4 \mathrm{~m}$ ATST (Advanced Technology Solar Telescope) and $4 \mathrm{~m}$ EST (European Solar Telescope) begin operation in the next decade. Meanwhile, the NST will be the largest off-axis telescope until the $8.4 \mathrm{~m}$ segments of the GMT (Giant Magellan Telescope) come on-line.

In contrast with most solar telescopes in operation which have on-axis configurations, the NST adopts a unique off-axis optical design. Since the Secondary Mirror (SM) and SM support structure in the off-axis configuration are placed to allow an unobstructed aperture, an off-axis solar telescope has advantages over the on-axis one: First, the off-axis design can vastly reduce stray light since there is no central obscuration, which reduces the telescope's Moduation Transfer Function at high spatial frequency. Second, more solid SM support structure can

W. Cao: E-mail: wcao@bbso.njit.edu, Telephone: 19735965301

Ground-based and Airborne Telescopes III, edited by Larry M. Stepp, Roberto Gilmozzi, Helen J. Hall Proc. of SPIE Vol. 7733, 773330 - (C) 2010 SPIE · CCC code: 0277-786X/10/\$18 - doi: 10.1117/12.856611 

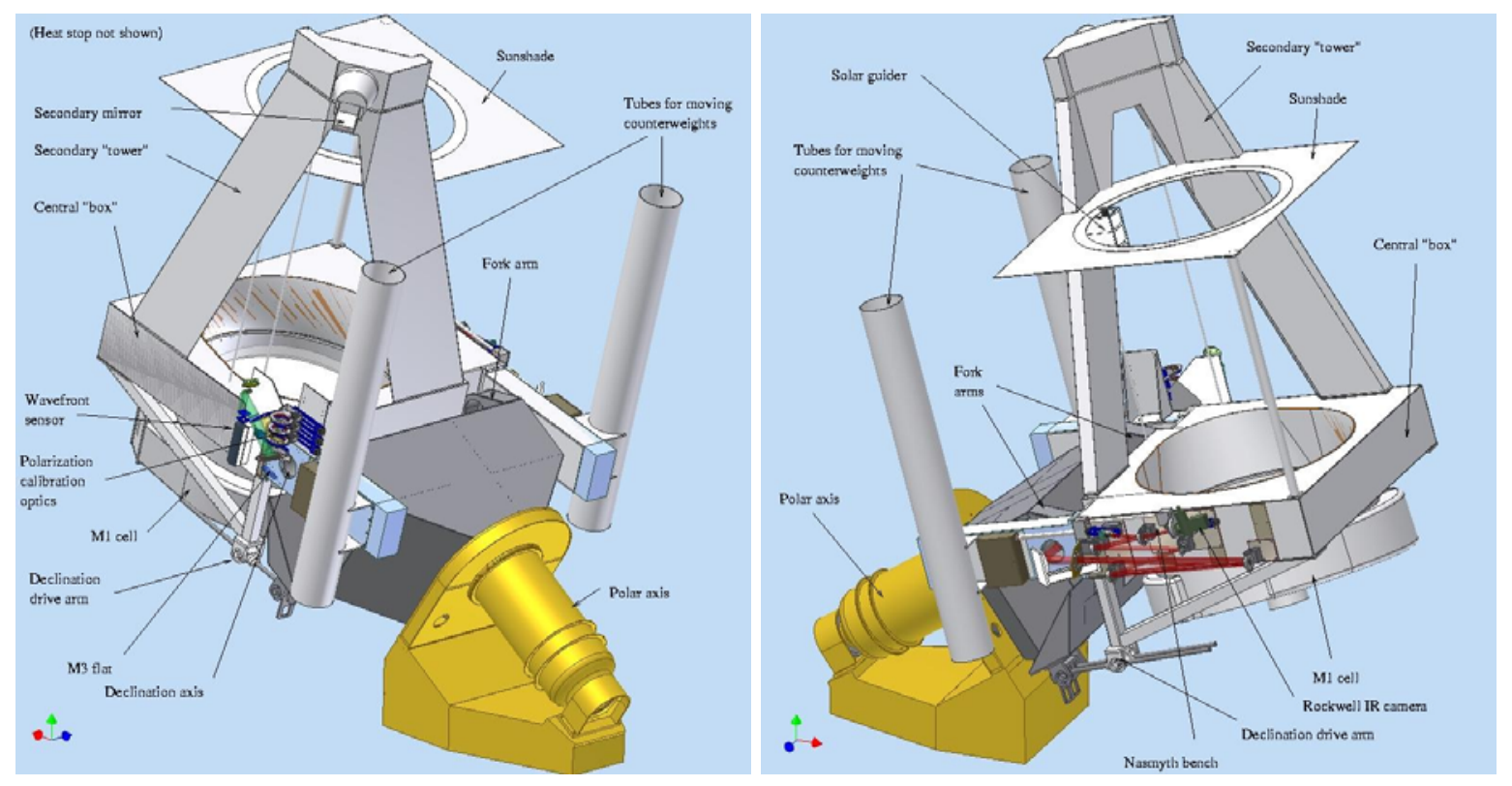

Figure 1. West (left panel) and east (right panel) view of $1.6 \mathrm{~m} \mathrm{NST}$.

be made to minimize vibrations and flexure without concern for its bulky size. Third, the prime focus field stop and heat reflector (heat-stop) are also configured off the solar beam. As a result, complicated cooling system and services have access to the heat-stop without obstructing the light path. Fourth, in an on-axis telescope configuration, the SM and SM support structure typically form a spider-shaped image on the telescope pupil. What is worse, this spider rotates during observations. The rotating spider in the pupil degrades the performance of the adaptive optics system. The off-axis telescope configuration offers an unobstructed pupil resolving this problem. Finally, in-situ cleaning and maintenance of the SM and heat-stop are much more easily done. On the other hand, the installation and alignment of a large off-axis telescope is much more challenging due to its asymmetric optical configuration. Benefitting from multiple advanced techniques and tools, the $1.6 \mathrm{~m}$ off-axis NST was successfully installed in Fall 2008 and a good alignment was accomplished in January 2009. First-light scientific observations have been attained at the Nasmyth focus and in the Coudé Lab. In this paper, we present a detailed description of installation and optical alignment of the NST. First-light observational results are also shown to demonstrate the validity of the NST optical alignment.

\section{NST CONFIGURATION}

The optical design of the NST and its scientific instrumentation have been described in detail by Goode et al. ${ }^{1,2}$ and Cao et al., ${ }^{3}$ respectively. Figure 1 shows the west and east views of the NST. The NST adopts an all reflecting, off-axis Gregorian configuration that consists of a parabolic primary mirror (PM or M1), prime focus field stop and heat reflector (heat-stop), elliptical secondary mirror (SM or M2) and diagonal flats (M3, M4, F3, M5, M6). The off-axis PM $(f / 2.4)$ is $1.7 \mathrm{~m}$ diameter with a clear aperture of $1.6 \mathrm{~m}$. The PM was made of Schott Zerodur with a extremely small coefficient of thermal expansion of $(0.0 \pm 1.0) \times 10^{-7} /{ }^{\circ} \mathrm{C}$. The Steward Observatory Mirror Lab. ${ }^{4}$ figured it to an accuracy of $16 \mathrm{~nm} \mathrm{rms}$ surface over the clear aperture. The $\mathrm{PM}$ has a radius of curvature of $7700 \mathrm{~mm}$ and a focal length of $3.85 \mathrm{~m}$. The $180^{\prime \prime}$ circular opening in the prime focus field stop defines the maximal unvignetted field of view (FOV) of the NST. The SM is an off-axis oblate ellipsoid (conic constant $=-0.83$ ) which has two conjugated foci. One focus of the SM is placed at the prime focus ( $\sim 300 \mathrm{~mm}$ away from the SM vertex) such that the light rays can be perfectly transferred to the other focus ( $\sim 6.49 \mathrm{~m}$ distant). The PM and SM together compose a classical Gregorian telescope system with an effective focal length of $83.2 \mathrm{~m}$ and a final $f$-ration of $f / 52$. Then the light is folded either by M3 and F4 through the declination axis to the Nasmyth focal plane on the east side of the telescope structure, or by M3 and M4 (flips up into the declination tunnel when needed) along the polar axis to the Gregory focal plane. Relay optics transfer 
light down to the Coudé Lab, two floors beneath the telescope deck. Figure 2 depicts the NST optical layout and a plan view of the parent mirror surfaces of the PM. Ignoring the aberrations caused by the residual error of the PM and SM surfaces, the final image quality of the NST predominantly depends on the position of the $\mathrm{SM}$ with respect to the PM. In the following sections, the detailed procedures of installation and alignment of the PM and SM are discussed.
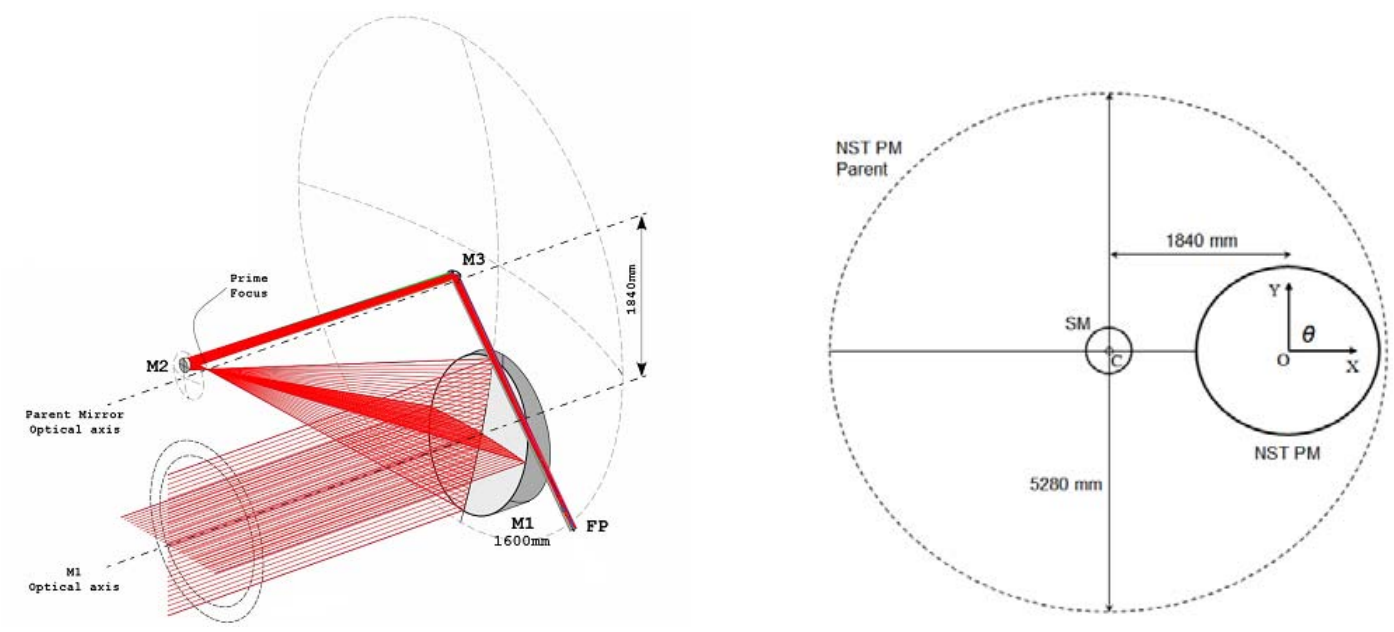

Figure 2. Left panel: NST optical layout with the parent mirrors of the PM (M1) and SM (M2). Right panel: End view of the NST pupils.

\section{PRIMARY MIRROR ALIGNMENT}

As illustrated in Figure 2, the NST PM is an off-axis segment of a $5.28 \mathrm{~m}, f / 0.729$ parabolic parent mirror. The focal length of the parent mirror is $3.85 \mathrm{~m}$. The distance $s$ between optical axes of the PM and its parent is $1.84 \mathrm{~m}$. The PM and its parent have a radius of curvature $R$ of $7.7 \mathrm{~mm}$ and a conic constant $k$ of -1 . Referring to the NST mechanical configuration shown in Figure 1, the PM optical axis should be vertical relative to the center section, and the symmetric plane has to be rotated about $48^{\circ}$ to place the optical beam just outside the west declination bearing. Although these parameters describe the system perfectly, they are not straightforward to measure in the telescope assembly and, thus, cannot realistically be used for alignment. To explicitly describe a rigid body, six degrees of freedom are essential. The right panel in Figure 2 shows the coordinate geometry: The origin is at the center of the PM. The $\mathrm{z}$ axis is perpendicular to the mirror surface and comes out of the page. Rotations of the PM about the $\mathrm{x}$-axis and $\mathrm{y}$-axis are defined as tilt $\left(0^{\circ}\right)$ and tilt $\left(90^{\circ}\right)$. Mirror rotation about the $\mathrm{z}$-axis is defined by $\theta$, with the convention of positive counterclockwise. The six degrees of freedom for the PM may be defined as: 1 . translations of the PM along axes: $\triangle x, \Delta y, \triangle z$ and 2. rotations of the PM about axes: tilt $\left(0^{\circ}\right)$, tilt $\left(90^{\circ}\right), \triangle \theta$.

\subsection{PM Alignment Theory}

The PM alignment is based on theoretical calculation of alignment aberrations relative to the position of the PM. Martin $^{5}$ derived the relation between them considering the Zernike coefficients of the surface error for tilt $\left(T_{0}\right.$, $\left.T_{90}\right)$, focus $F$, coma $\left(C_{0}, C_{90}\right)$ and astigmatism $\left(A_{0}, A_{45}\right)$. The polynomials are defined such that the surface displacement is

$$
\delta z=T_{0} \rho \cos \theta+T_{90} \rho \sin \theta+F\left(2 \rho^{2}-1\right)+A_{0} \rho^{2} \cos 2 \theta+A_{90} \rho^{2} \sin 2 \theta+C_{0}\left(3 \rho^{3}-2 \rho\right) \cos \theta+C_{90}\left(3 \rho^{3}-2 \rho\right) \sin \theta,
$$

where $\rho=r / a$ is the normalized radius on the PM $(a=0.85 \mathrm{~m})$. After nulling off-axis aberrations of the PM itself, alignment aberrations are seen when the PM is displaced from the ideal position. Martin ${ }^{5}$ derived the PM aberrations as a function of misalignment parameters: $T_{0}=-a \triangle x / R, T_{90}=-a \triangle y / R, F=-a^{2} \triangle z /\left(4 R^{2}\right)-$ 
$k a^{2} s \triangle x /\left(2 R^{3}\right), C_{0}=-k a^{3} \triangle x /\left(6 R^{3}\right), A_{0}=-k a^{2} s \triangle x /\left(2 R^{3}\right), C_{90}=k a^{3} s \triangle \theta /\left(6 R^{3}\right)-k a^{3} \triangle y /\left(6 R^{3}\right), A_{45}=$ $k a^{2} s^{2} \triangle \theta /\left(2 R^{3}\right)-k a^{2} s \triangle y /\left(2 R^{3}\right)$.

The Steward Observatory Mirror Lab measured the PM alignment aberrations with aid of a modified interferometer and a null corrector consisting of a computer-generated hologram (CGH) and a spherical mirror. ${ }^{5,6}$ The ideal position of the PM relative to the prime focus were carefully determined by minimizing the alignment aberrations which were measured with the interferometer and CGH based null corrector.

\subsection{Alignment with Laser Tracker System}

Based on the PM alignment theory and interferometric test, a commercial laser tracker (FARO Tracker X) was employed during the installation and alignment of the PM. Laser tracker is a portable coordinate measuring machine which uses laser feed-back and motorized steering mirrors to track the location of a Spherical Mounted Retroreflector (SMR). The absolute radial distance between SMRs and tracker can be measured with an extremely high precision of $\pm 10 \mu \mathrm{m} / \mathrm{m}$. Software can record 3 dimensional locations of SMRs in a variety of coordinate system. As a result, it is particularly suitable for optical alignment and metrology.

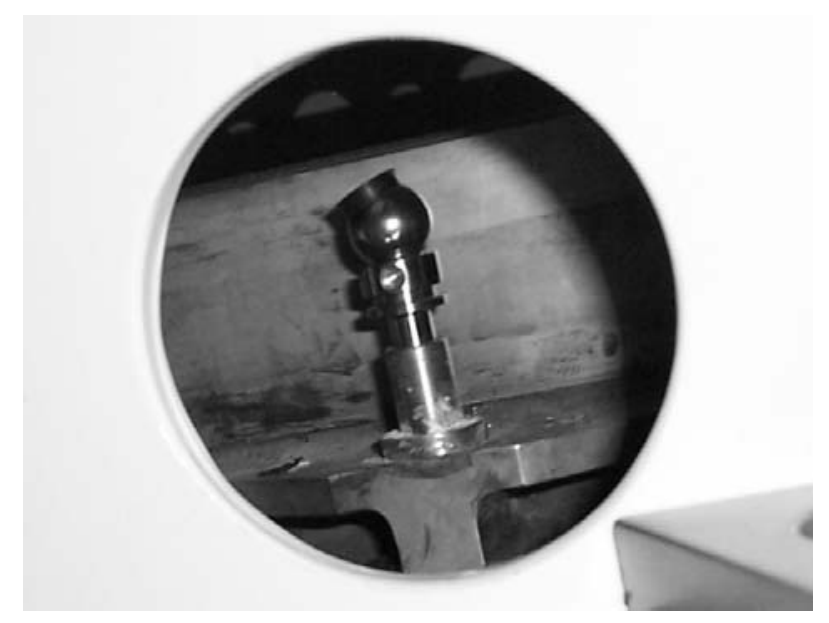

Figure 3. One fiducial attached to the PM tangential puck for the laser tracker measurement. The gimbal is a a spherical mounted retroreflector. There are four fiducials attached around the PM.

Once the aforementioned interferometric measurements of alignment aberrations were completed in the Mirror Lab, four fiducials were attached around the PM. The fiducial consists of a SMR and its nest mounted on the PM tangential puck. Figure 3 shows one example of a fiducial. These fiducials were precisely measured relative to the optical axis and prime focus using the laser tracker system at the Mirror Lab following the conclusion of the figuring. The obtained data were used for subsequently locating the PM in the field.

ATT Metrology Services was contracted to measure the fiducials when the PM was installed. Four SMRs were installed in their nests. A laser tracker was mounted one point in the dome where it had view for all four SMRs. After the calibration of relative positions, absolute radial distances between the laser tracker and four fiducials were precisely determined based on previously obtained data in Mirror Lab. Monitoring the measurements of laser tracker, the PM was tweaked to place the prime focus of the PM within a $1 \mathrm{~mm}$ box centered in the prime focus stop. Once this procedure was completed, the PM is fixed mechanically.

\section{HEAT STOP ADJUSTMENT}

Figure 4 shows prime focus field stop and heat reflector (Heat Stop). Two interchangeable field stops are available for the NST prime focus. The field stop in operation has an opening of $3.8 \mathrm{~mm}$ defining a unvignetted circle FOV of $180^{\prime \prime}$. The heat reflector is silvered to maximize reflectance for unwanted solar beam. Internal cooling system and air-knife further limit heating at the SM complex to an acceptable lever. 


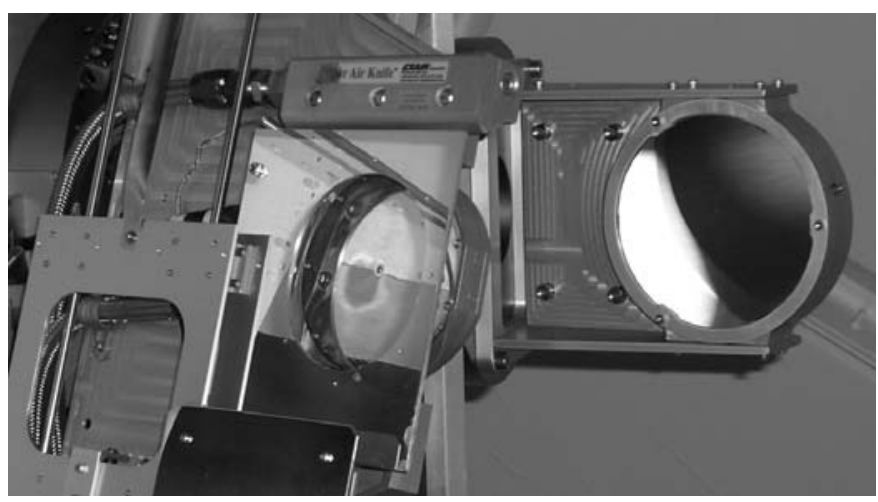

Figure 4. The NST prime focus field stop and heat reflector in front of the NST secondary mirror.

The PM has been tweaked to place its prime focus within a $1 \mathrm{~mm}$ box centered in the prime focus stop. However, this accuracy is too coarse to position the heat stop. Fine-tuning is essential in order to minimize alignment aberrations for high resolution observations. Figure 5 shows the spot diagram over a FOV of $180^{\prime \prime}$ at the prime focus. The prime focus is highly aberrated beyond the center of FOV. However, this provides a good measure of the prime focus position allowing adjustment of the heat stop with respect to the PM.

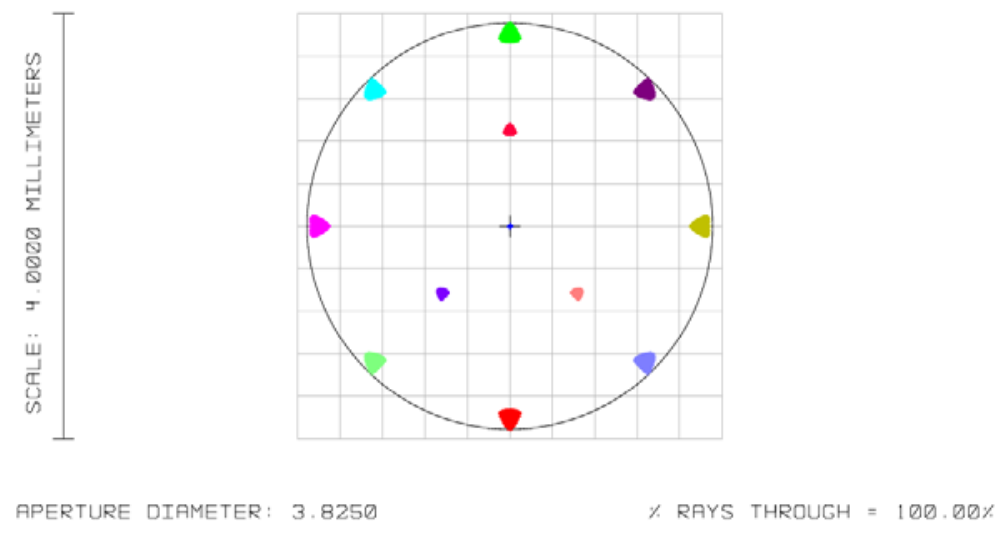

Figure 5. Spot diagram over a FOV of $180^{\prime \prime}$ at the prime focus.

With the PM fixed, a white sheet with measured grid was placed at prime with its center aligned to the heat stop. The sheet was imaged with a video camera which was mounted near the prime focus. On good seeing nights, a sky area with a cluster of stars were chosen to be imaged on the sheet at the prime. The heat stop was then moved around to determine the best position of the optical axis by putting a star with a minimized aberration at field stop. The focal plane were measured using a wavefront sensor (WFS), which is discussed in subsequent sections.

\section{SECONDARY MIRROR ALIGNMENT}

During the first attempt of the PM alignment, the SM was roughly positioned using manual measurements to the field stop. Having fixed the position of the PM, and having the SM close to the correct position, the WFS can be used to fine tune the SM position. The problem with this method is that there is not a good way to separate the effects of clocking errors from the those of the tip/tilt errors using the measured wavefront. Ultimately, the method failed. 


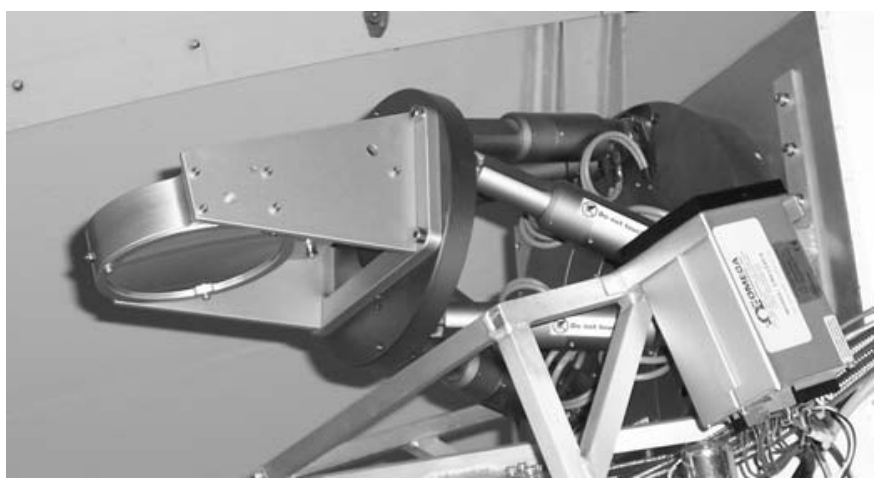

Figure 6. The NST SM mounted on a PI hexapod.

\subsection{Alignment with Laser Tracker}

During the polishing of the PM, Steward Observatory was contracted to measure the NST SM. These tests confirmed proper shape for the mirror surface. At the same time laser tracker measurements were made relating tracker balls mounted in permanent nests on the SM mount to the actual position of the mirrors foci.

\subsection{SM Motion}

As shown in Figure 6, the SM is mounted on a PI hexapod allowing displacements around 2 points for telescope alignment: 1. Displacement around the Prime Focus minimizes the angle between the optical axis of both PM and SM. This impacts the pointing and the field astigmatism with a magnitude changing linearly with the field angle. 2. Displacement around the Center of Curvature of SM impacts coma and astigmatism with a magnitude constant over the field. Figure 7 shows spot diagrams of NST with SM rotated about prime focus and about its center of curvature, respectively.
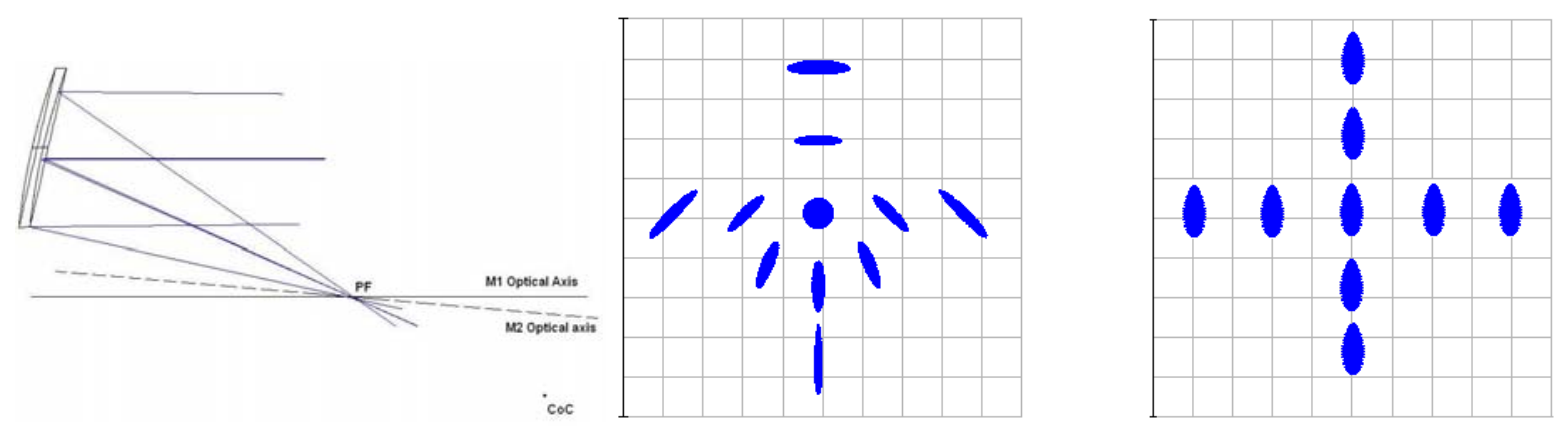

Figure 7. Left panel: NST SM degrees of freedom. Middle panel: Spot diagram of NST with SM rotated about prime focus showing astigmatism. Right panel: Spot diagram of NST with SM rotated about its center of curvature showing coma.

\subsection{Wavefront Sensor Measurement}

Stellar wavefront measurements were made using Wavefront Sciences CLAS-2D Sensor. The SM is then moved to minimize aberrations or, in a closed loop system with an active PM, the SM is moved to minimize force applied to the PM. Poor stellar image quality made this method difficult to use although that is likely due to the lake environment. Wavefront Sciences produced a correlation tracking algorithm which can be use for the SM adjustment using the sun but sending sunlight to the WFS proved problematic for several reasons and the decision was made to wait for the $\mathrm{AO}$ system to come online. 


\section{FIRST LIGHT OBSERVATIONAL RESULTS}

The largest solar telescope, the $1.6 \mathrm{~m}$ off-axis NST has successfully been installed and aligned at the BBSO. First light observations have been carried out at the Nasmyth focus ${ }^{7}$ and in the Coudé Lab and revealed a number of exciting scientific results. ${ }^{8} 9$ Figure 8 shows two samples of observations with the NST. These observations also demonstrate the validity of the NST optical alignment. Current efforts focus on the development of post-focus instrumentations, the implementation of the NST active optics, and the upgrading of the WSF. The experience obtained in installation and alignment will be benefiting to the next generation solar telescopes, such as ATST and EST.
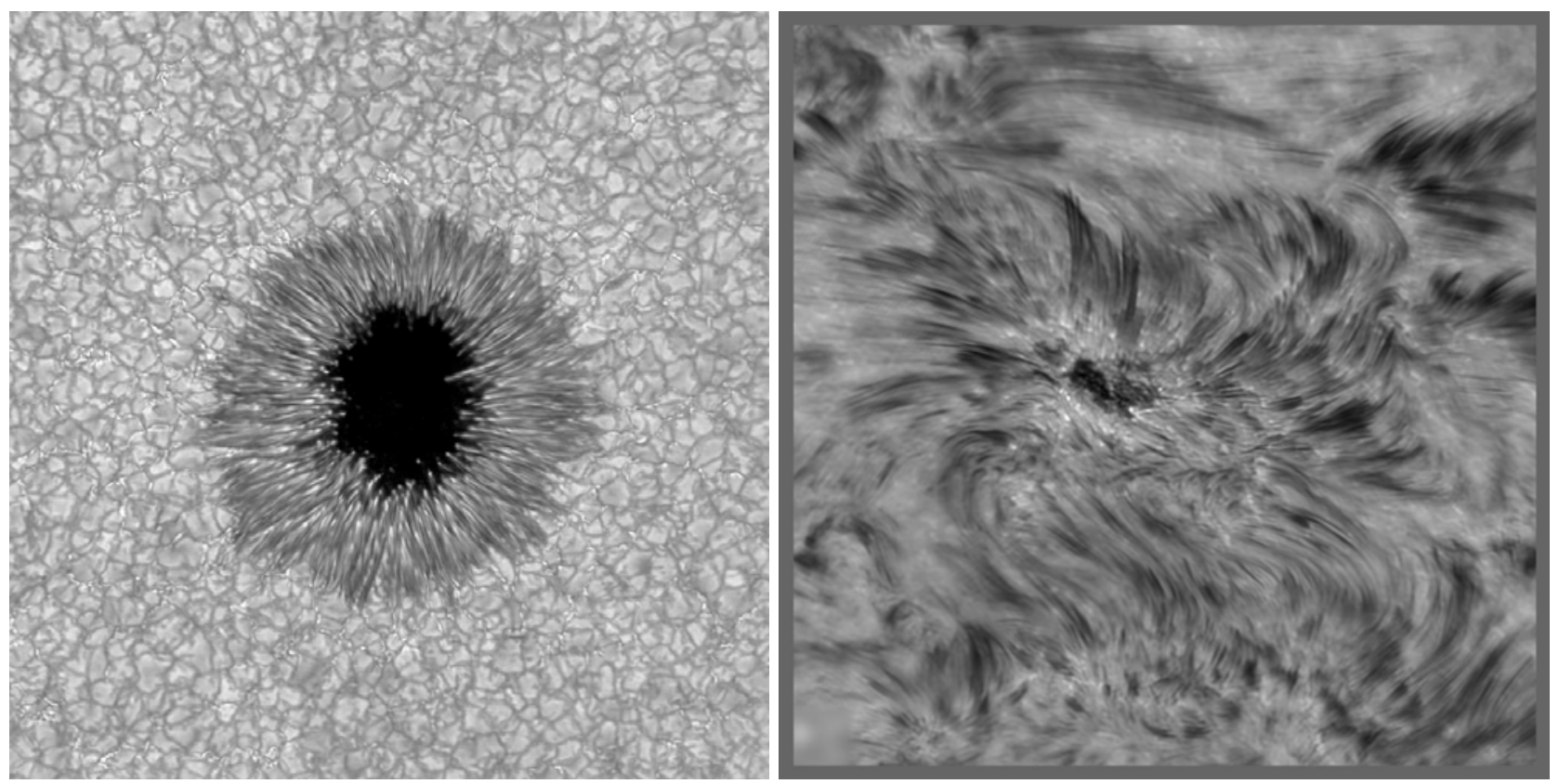

Figure 8. Left panel: Sunspot observed in TiO $706 \mathrm{~nm}$ on July 2, 2010. Right panel: An active region observed in Ha blue wing on June 14, 2010.

\section{ACKNOWLEDGMENTS}

We would like to thank Buddy Martin of Steward Observatory Mirror Lab. for his help in alignment of the NST PM and SM. This work is supported by the NSF grants AGS-0847126 and AGS-0745744, the AirForce grant AFOSR-FA9550-09-1-0655 and by the NASA grant NASA-NNX08BA22G.

\section{REFERENCES}

[1] Goode, P. R., Denker, C., Didkovsky, L. I, Kuhn, J. R., and Wang, H., "1.6 M Solar Telescope in Big Bear - The NST," Journal of Korean Astron. Soc. 36, 125-133 (2003).

[2] Goode, P. R., Coulter, R., Gorceix, N., Yurchyshyn, V., and Cao, W., "The NST: First results and some lessons for ATST and EST," Astron. Nachr. 331, 620-623 (2010).

[3] Cao, W., Gorceix, N., Coulter, R., Ahn, K., Rimmele, T. R., and Goode, P. R., "Scientific Instruments of 1.6 m New Solar Telescope in Big Bear," Astron. Nachr. 331, 636-639 (2010).

[4] Martin, B, [Fabrication and Testing of the NST Primary Mirror], Steward Observatory Mirror Lab (2008).

[5] Martin, B, [Guide to Aligning the NST Mirror for an Optical Test], Steward Observatory Mirror Lab (2005).

[6] Zhao, C., Zehnder, R, Burge, H. J., and Martin, M. H., "Testing an Off-axis Parabola with a CGH and a Spherical Mirror as Null Lens," Proc. SPIE 5869, 261-272 (2005). 
[7] Cao, W., Gorceix, N., Coulter, R., Wöger, F., Ahn, K., Shumko, S., Varsik, J., Coulter, A., and Goode, P. R., "Nasmyth focus instrumentation of the New Solar Telescope at Big Bear Solar Observatory," Proc. SPIE 7735, 217-223 (2010).

[8] Goode, P. R., Yurchyshyn, V., Cao, W., Abramenko, V., Andic, A., Ahn, K., and Chae, J., "Highest resolution observations of the quietest Sun," ApJ 714, L31-L35 (2010).

[9] Cao, W., Ning, Z., Goode, P. R., Yurchysyn, V., and Ji, H., "Evidence of filament upflows orignating from intensity oscillations on the solar surface," ApJ 719, L95-L98 (2010). 